\title{
A Comparison of Frequentist and Bayesian Model Based Approaches for Missing Data Analysis: Case Study with a Schizophrenia Clinical Trial
}

\section{Supplemental Materials for Online}

1. Sample Program Codes for Selected Bayesian Models

a) MMRM model

The follow codes are used to fit the MMRM model with SAS proc MCMC. The input dataset "forMC1" contain variables of change from baseline PANSS: y1, y2, ..., y5 (missing data are indicated as .), base (standardized baseline PANSS), trtn (treatment indicator, 0 for placebo, 1 for test, 2 for active).

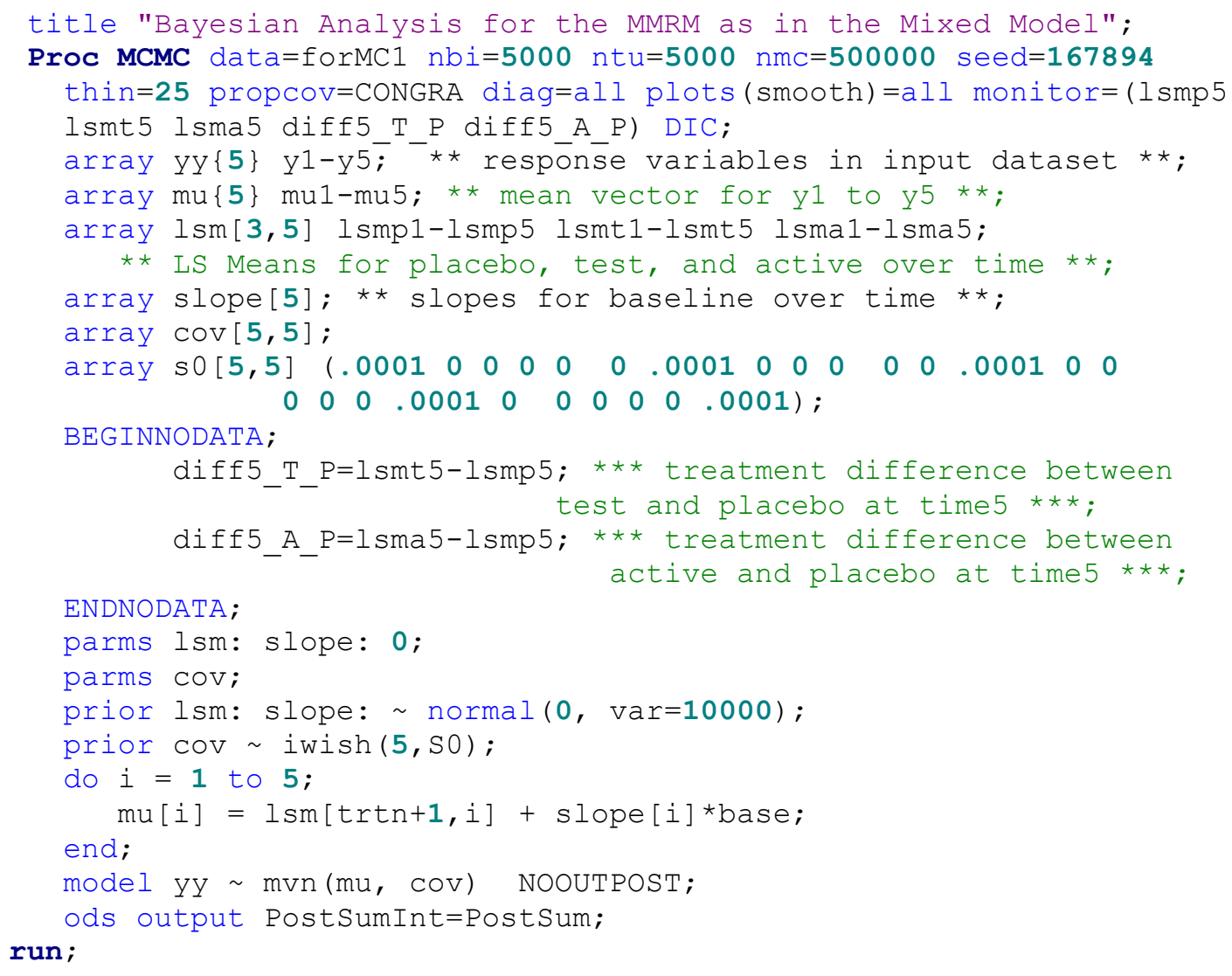

b) Selection model (SAS proc MCMC) 
The following codes will fit a selection model between test drug and placebo as described in Section 3.2 and 4.2. The dataset contains same variables as above plus the dropout time variable:

Drop $=1$ if not dropout, $j(>1)$ if dropout at time $j$.

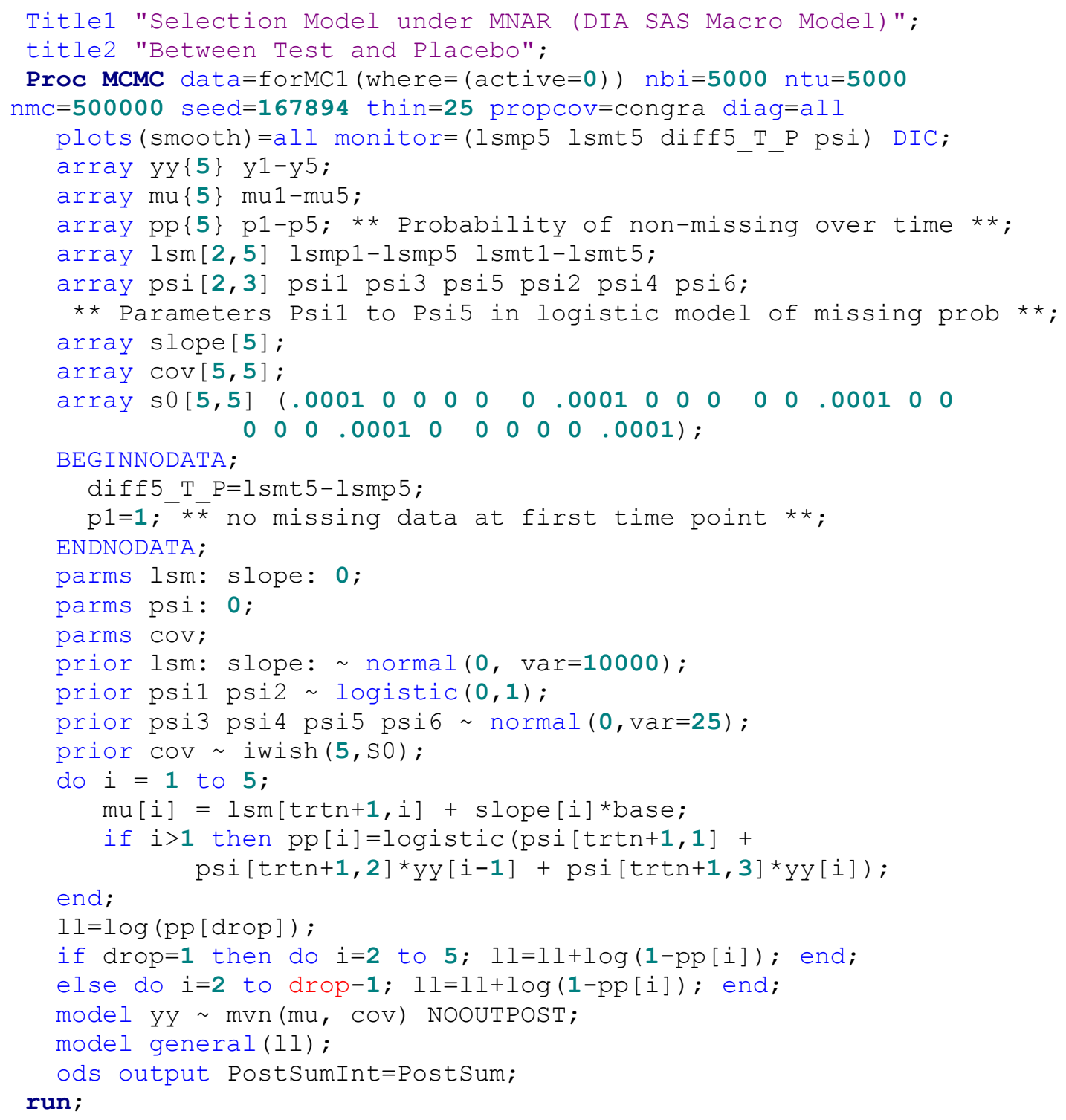

c) Selection model with modified dropout probability (SAS proc MCMC) The following codes will fit the selection model for all three treatment groups with modified missing data probability model as described in Section 4.3.

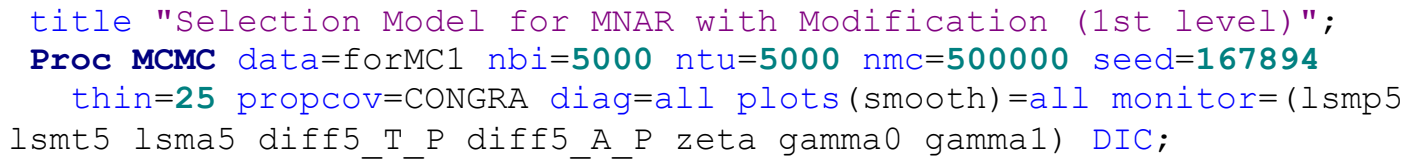




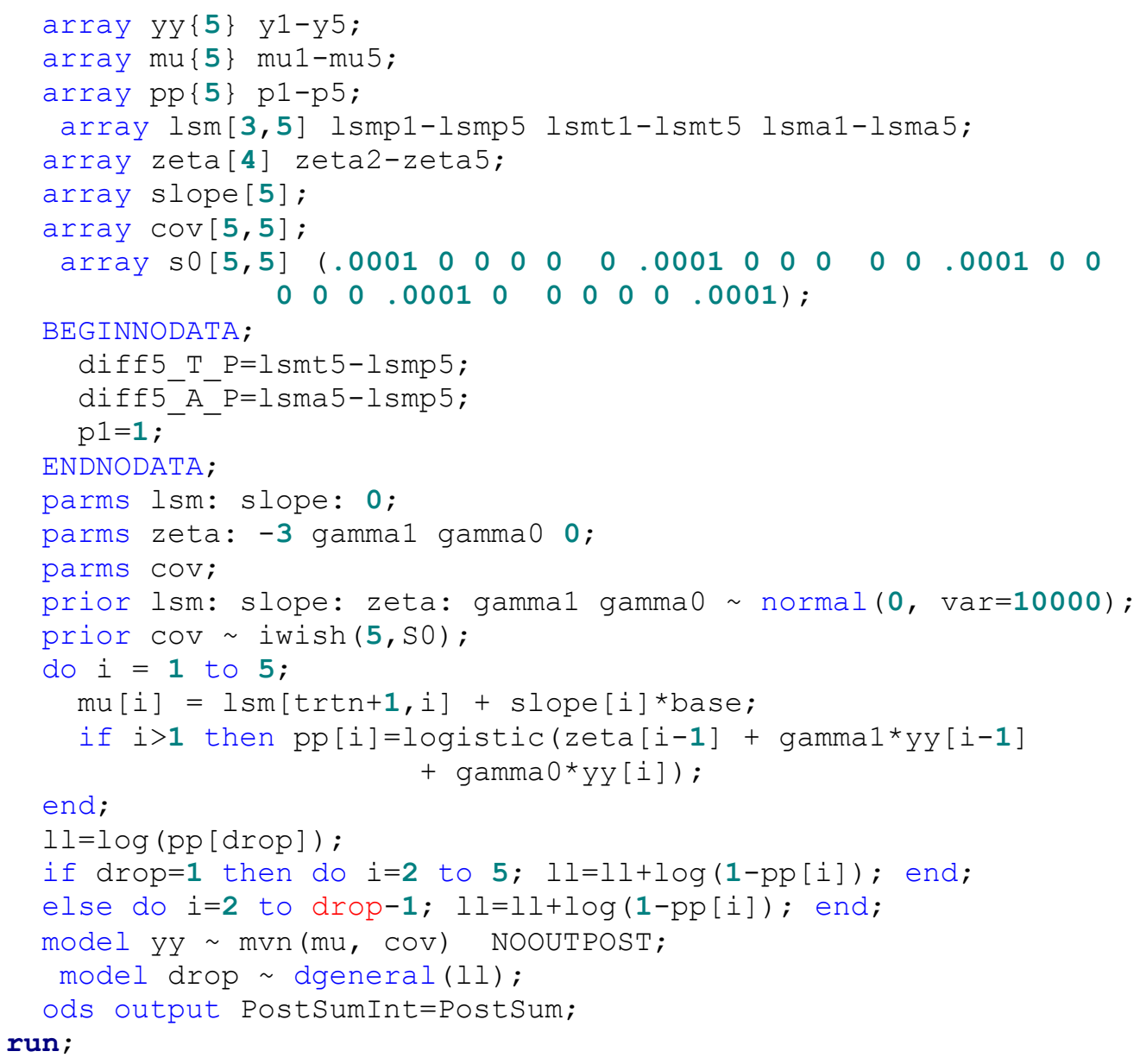

d) Selection model with R and Stan

The following codes will fit a selection model between active and placebo as described in Section 3.2 and 4.2.

library(coda)

library(rstan)

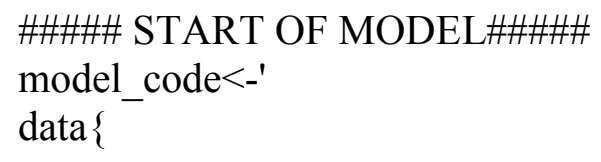




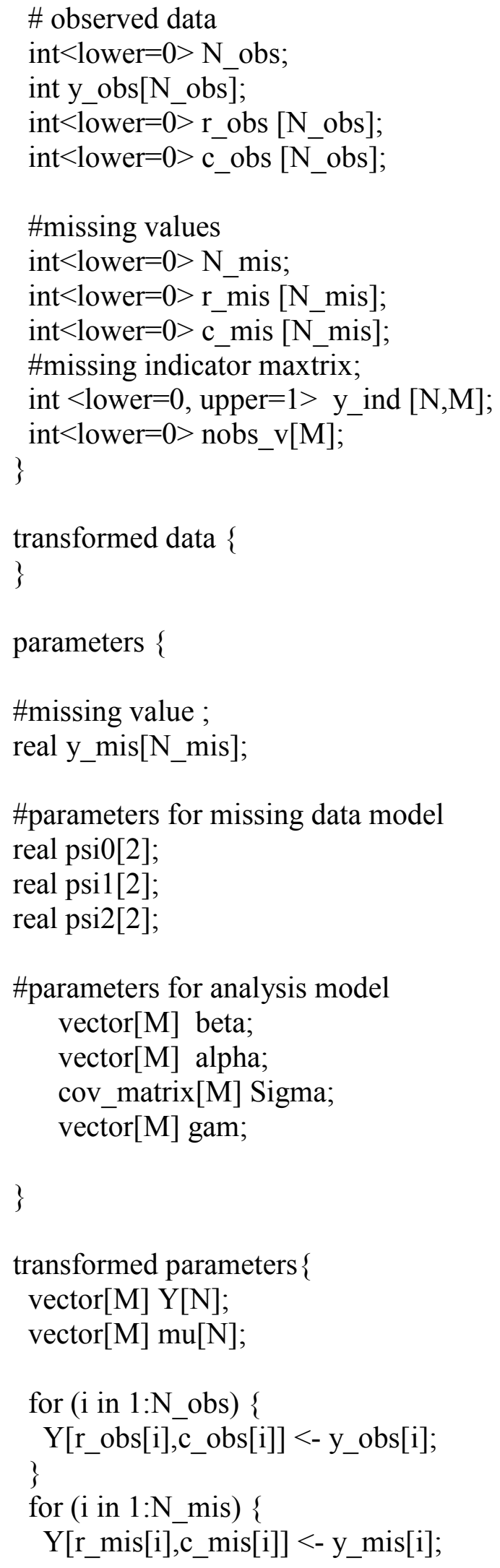




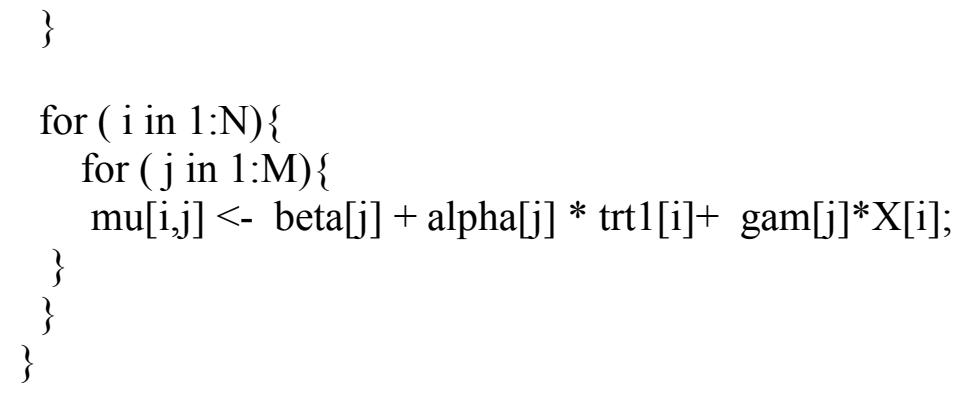


wd<-"H:/research/missingdata/out/dia/output/mnar_sel_2trt_active1"

if (!exists(wd)) dir.create(wd,recursive $=\mathrm{T})$

setwd(wd)

rimagefile $<-$ "sel_2trt_active4Surv_summary.Rdata"

statsfile <-"sel_2trt_active4Surv_summary.csv"

pdffile<-"sel_2trt_active4Surv_plots.pdf"

\#data preparation

d<-read.csv("H:/research/missingdata/data/Schizophrenia_wide.csv")

$\mathrm{d}<-\mathrm{d}[\mathrm{d} \$$ trt \%in\% c("Placebo", "Active"), ]

$\mathrm{y}<-\mathrm{d}[$,paste("PANSS.",0:5, sep="")]

$\mathrm{N}<$-nrow(d) \#number of subjects

$\mathrm{M}<-5$ \#number of visits

$\mathrm{S}<-\operatorname{diag}(\mathrm{M})$

$\mathrm{y}<-\mathrm{y}[, 2: 6]-\mathrm{y}[, 1]$

loc_mis $<$-which( is.na(y),arr.ind $=\mathrm{T}$ )

loc_obs<-which(! is.na(y),arr.ind=T)

r_mis $<$-as.numeric(loc_mis[,1])

c_mis $<$-as.numeric(loc_mis[,2])

r_obs<-as.numeric(loc_obs[,1])

c_obs $<$-as.numeric $\left(10 c \_o b s[, 2]\right)$

y_obs $<-$ y[loc_obs]

ymean $<$-mean(y_obs)

y_mis<-y[loc_mis]

N_obs=length(y_obs)

N_mis=length(y_mis)

\#covariates

bl<-d\$PANSS.0 -mean(d\$PANSS.0)

$\mathrm{X}<$-as.numeric(bl);

$\operatorname{trt} 1<-(\mathrm{d}$ \$trt=="Active" $) * 1.0001$

trt $<$-as.numeric(factor(d\$trt, levels=c("Placebo", "Active") , ordered=T))

y_ind $1<-$ is.na $(y)^{*} 1$;

$\mathrm{y}$ _ind $<-$ matrix $\left(\operatorname{as.numeric}\left(\operatorname{unlist}\left(\mathrm{y} \_\right.\right.\right.$ind 1$\left.)\right), \mathrm{nrow}=\mathrm{nrow}\left(\mathrm{y} \_\right.$ind 1$\left.)\right)$

nobs_v<-as.numeric(nrow(d)-apply(y_ind,2,sum))

stan_dat<-list("c_obs"=c_obs,"r_obs"=r_obs,"y_obs"=y_obs,

"N"=N,"M"=M, "trt1"=trt1,"trt"=trt,"ymean"=ymean,

"X"=X, "S"=S, "y_ind"=y_ind, nobs_v"=nobs_v,

"N_obs"=N_obs, "N_mis"=N_mis, "r_mis"=r_mis, "c_mis"=c_mis) 


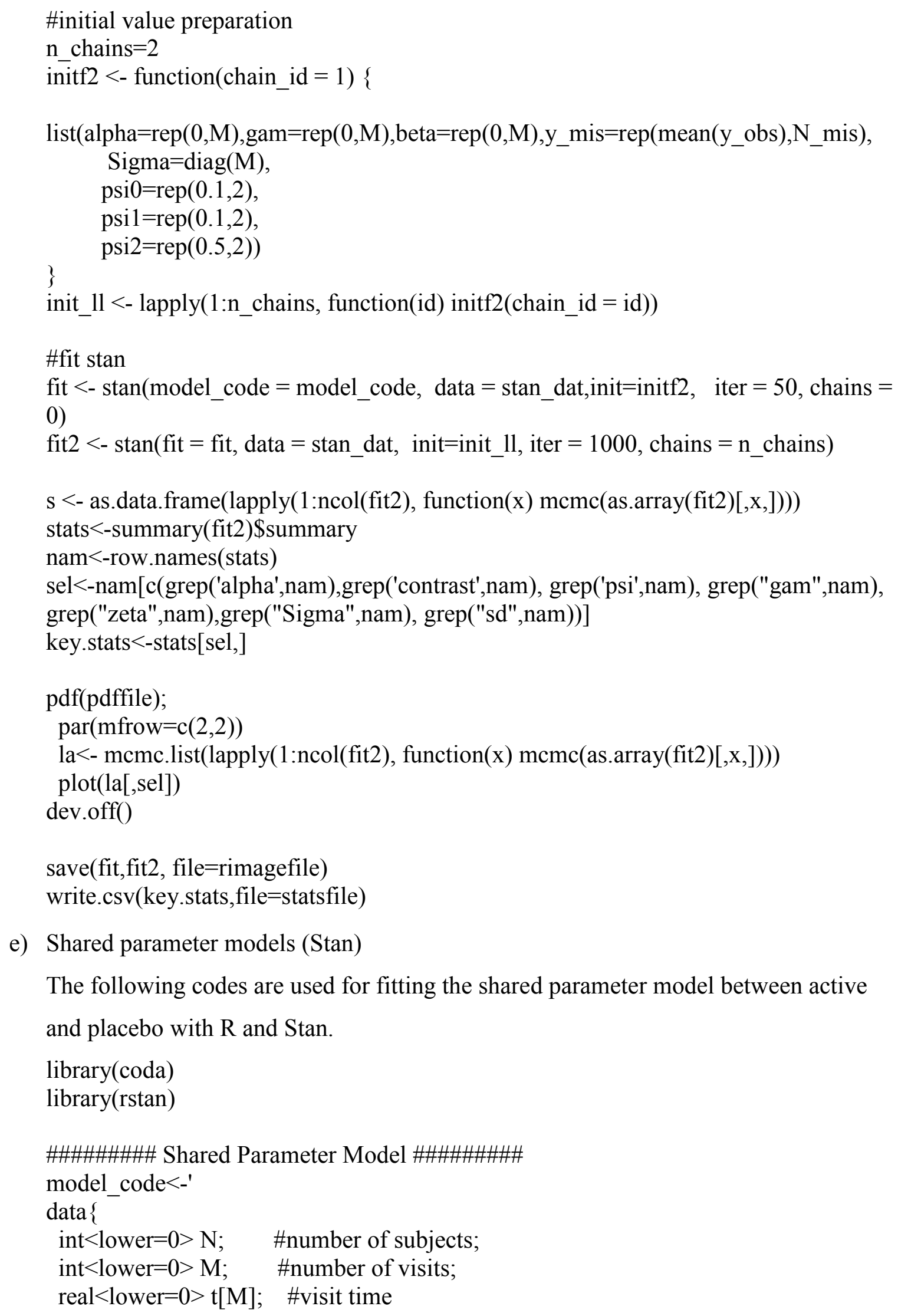




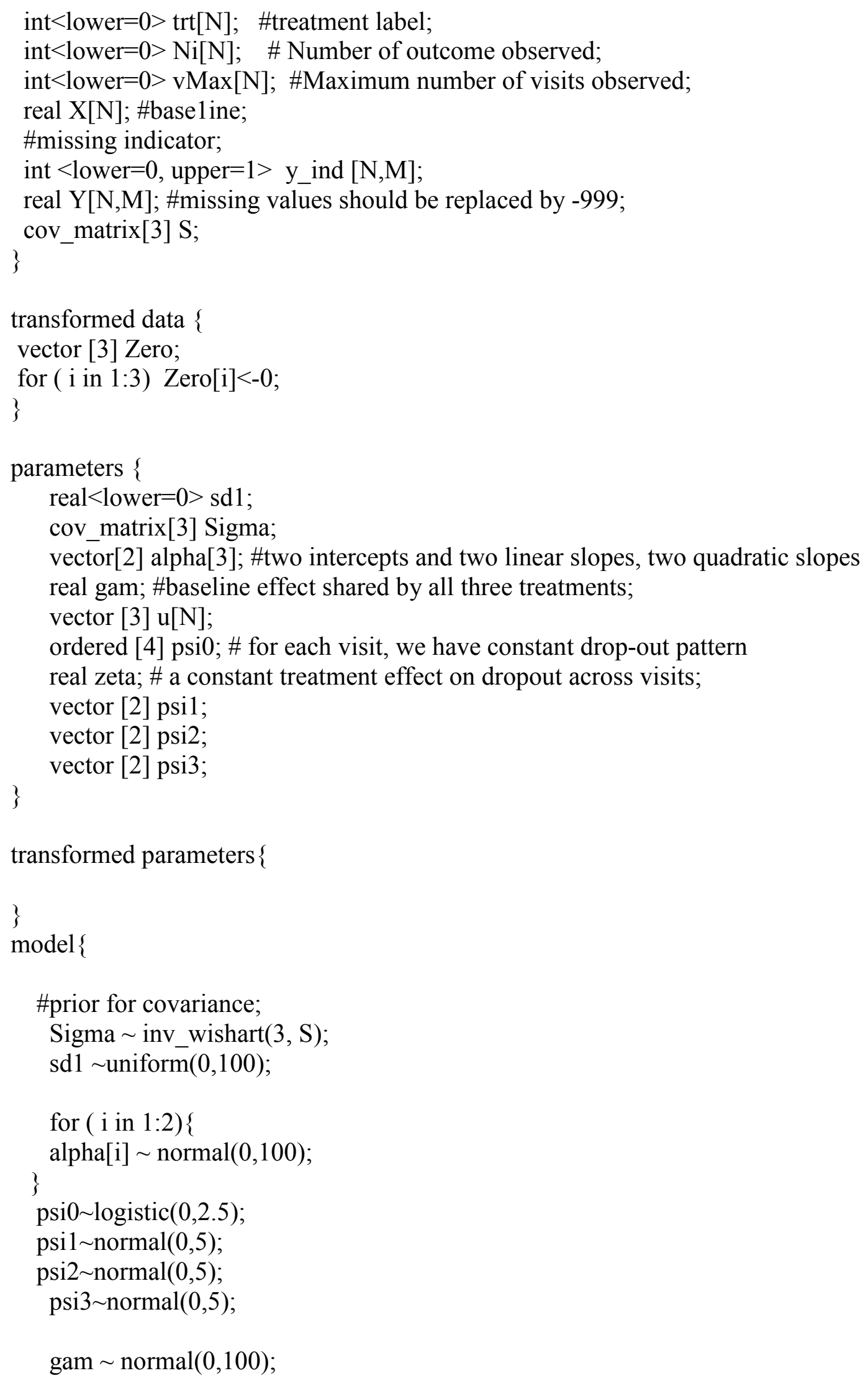




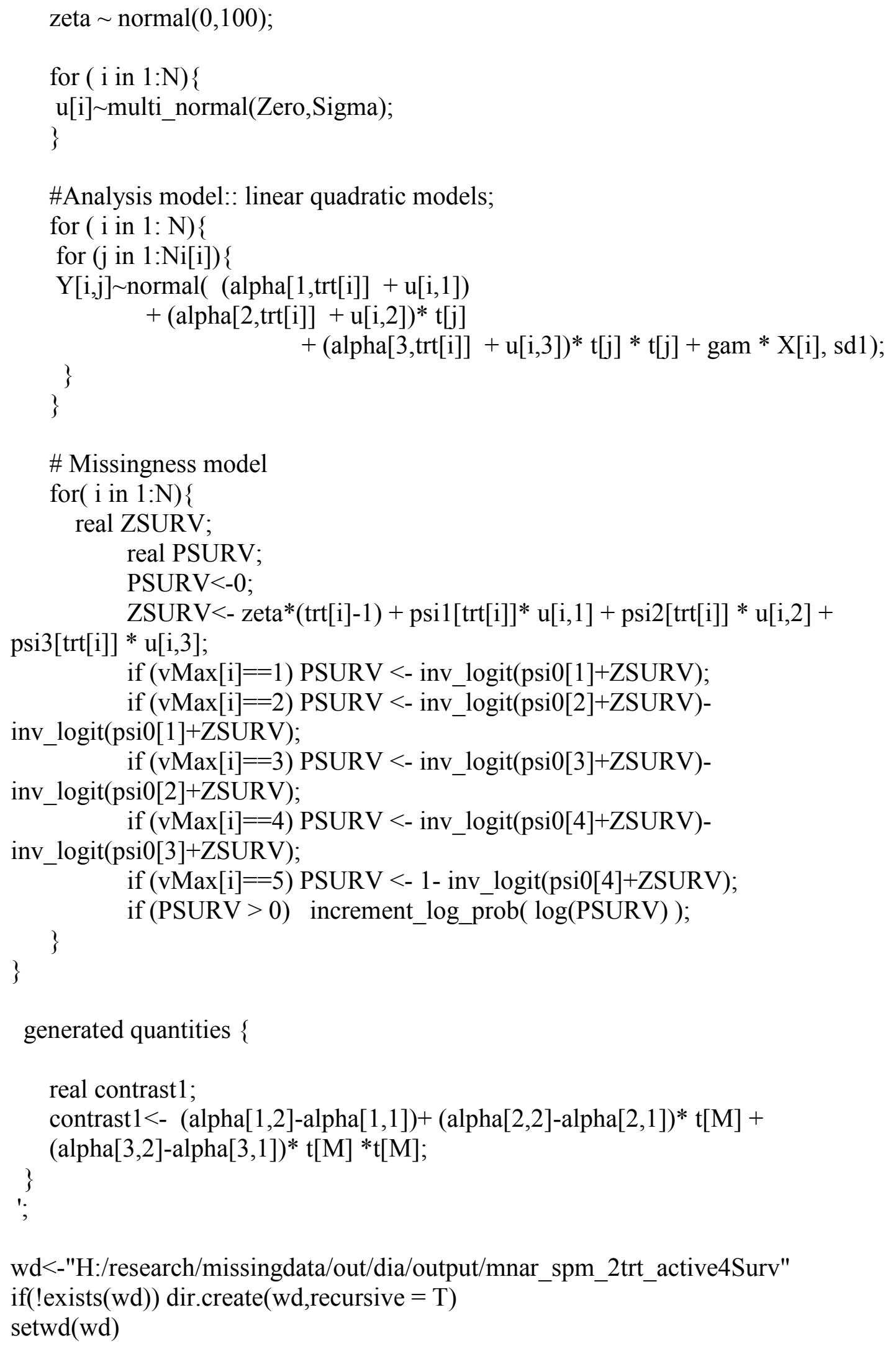


rimagefile <- "spm_2trt_active4Surv_summary.Rdata" statsfile <-"spm_2trt_active4Surv_summary.csv"

pdffile<-"spm_2trt_active4Surv_plots.pdf"

d<-read.csv("H:/research/missingdata/data/Schizophrenia_wide.csv")

\#focus on two treatments:

$\mathrm{d}<-\mathrm{d}[\mathrm{d}$ \$trt \%in\% c("Placebo", "Active"),]

$\mathrm{y}<-\mathrm{d}[$,paste("PANSS.",0:5, sep="")]

$\mathrm{N}<$-nrow(d) \#number of subjects

$\mathrm{M}<-5$ \#number of visits

$\mathrm{y}<-\mathrm{y}[, 2: 6]-\mathrm{y}[, 1]$

$\mathrm{y}<$-matrix(as.numeric(unlist(y)),nrow $=\operatorname{nrow}(\mathrm{y}))$

\#covariates

bl<-d\$PANSS.0 -mean(d\$PANSS.0)

$\mathrm{X}<$-as.numeric(bl);

trt $<$-as.numeric(factor(d\$trt, levels=c("Placebo","Active"), ordered=T))

$\mathrm{t}<$-c $(4,7,14,21,28) / 7$

y_ind $1<-$ is.na $(y) * 1$;

y_ind $<-$ matrix(as.numeric(unlist(y_ind1)),nrow=nrow(y_ind1))

$\overline{\mathrm{Ni}}<-5$-apply(y_ind, 1, sum $)$;

$\operatorname{vMax}<-\operatorname{sapply}(\mathrm{Ni}$, function(x) $\min (5, \mathrm{x}+1))$;

vMax $<-\mathrm{Ni}$;

$\mathrm{Y}<-\mathrm{t}(\operatorname{apply}(\mathrm{y}, 1$,function(x) sapply(x,function(xx) $\{$ ifelse(is.na(xx),-999,xx) $\}))$ )

$\mathrm{S}<-\operatorname{diag}(3)$

stan_dat<-list("N"=N,"M"=M,'Y'=Y, "y_ind"=y_ind,

"Ni"=Ni,"vMax"=vMax,"trt"=trt, "X"=X,'t'=t, "S"=S)

\# generate a list of lists to specify initial values

n_chains $<-2$

initf $<$ - function(chain_id $=1)\{$

list(alpha $=$ matrix $(0,3, \overline{2})$,gam $=0$,

$\operatorname{Sigma}=\operatorname{diag}(3), \mathrm{u}=\operatorname{matrix}(0, \mathrm{~N}, 3$, byrow $=\mathrm{T})$,

$\mathrm{sd} 1=1$,

$\mathrm{psi} 0=\mathrm{c}(0.1,0.2,0.3,0.4)$,

psi $1=\mathrm{c}(0.5,0.5)$,

psi2 $=\mathrm{c}(0.5,0.5), \mathrm{psi} 3=\mathrm{c}(0.5,0.5), \mathrm{zeta}=0.1)$

\}

init_1l <- lapply(1:n_chains, function(id) initf2(chain_id=id)) 


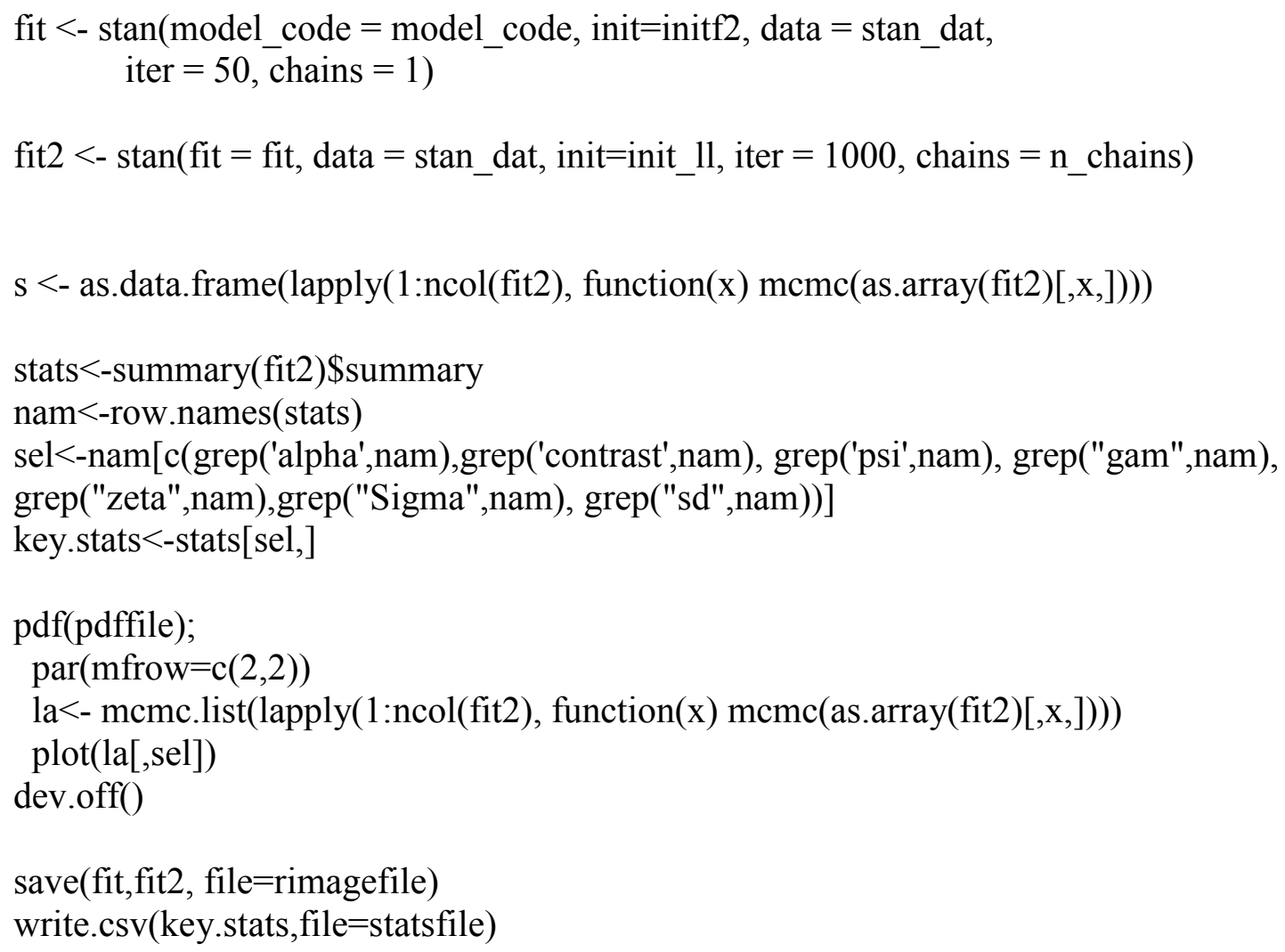


2 Trace and Density Plots for Selected Parameters from the Bayesian Selection Models

a). Trace plot and density plots for the selection model with modified parameters (based on SAS PROC MCMC codes as described in (c) above). The model parameters from top to bottom are the treatment contrast between test drug and placebo (diff5_T_P), between active and placebo (diff5_A_P), the intercept terms $\left\{\zeta_{2}, \zeta_{3}, \zeta_{4}, \zeta_{5}\right\}, \gamma_{0}$ (i.e., the regression coefficient on $Y_{j}$ ), and $\gamma_{1}$ (i.e., the regression coefficient for $Y_{j-1}$ ), respectively.

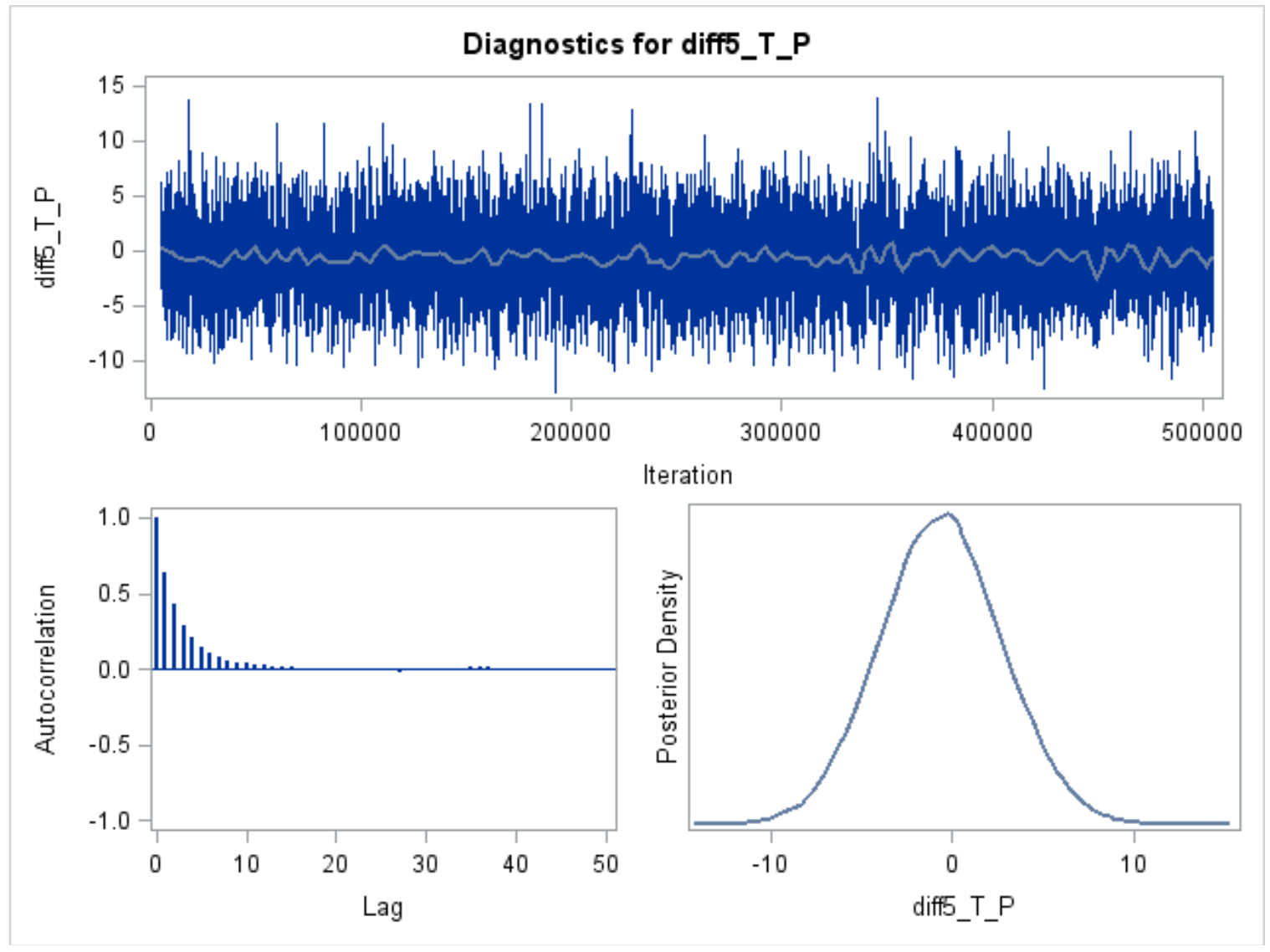




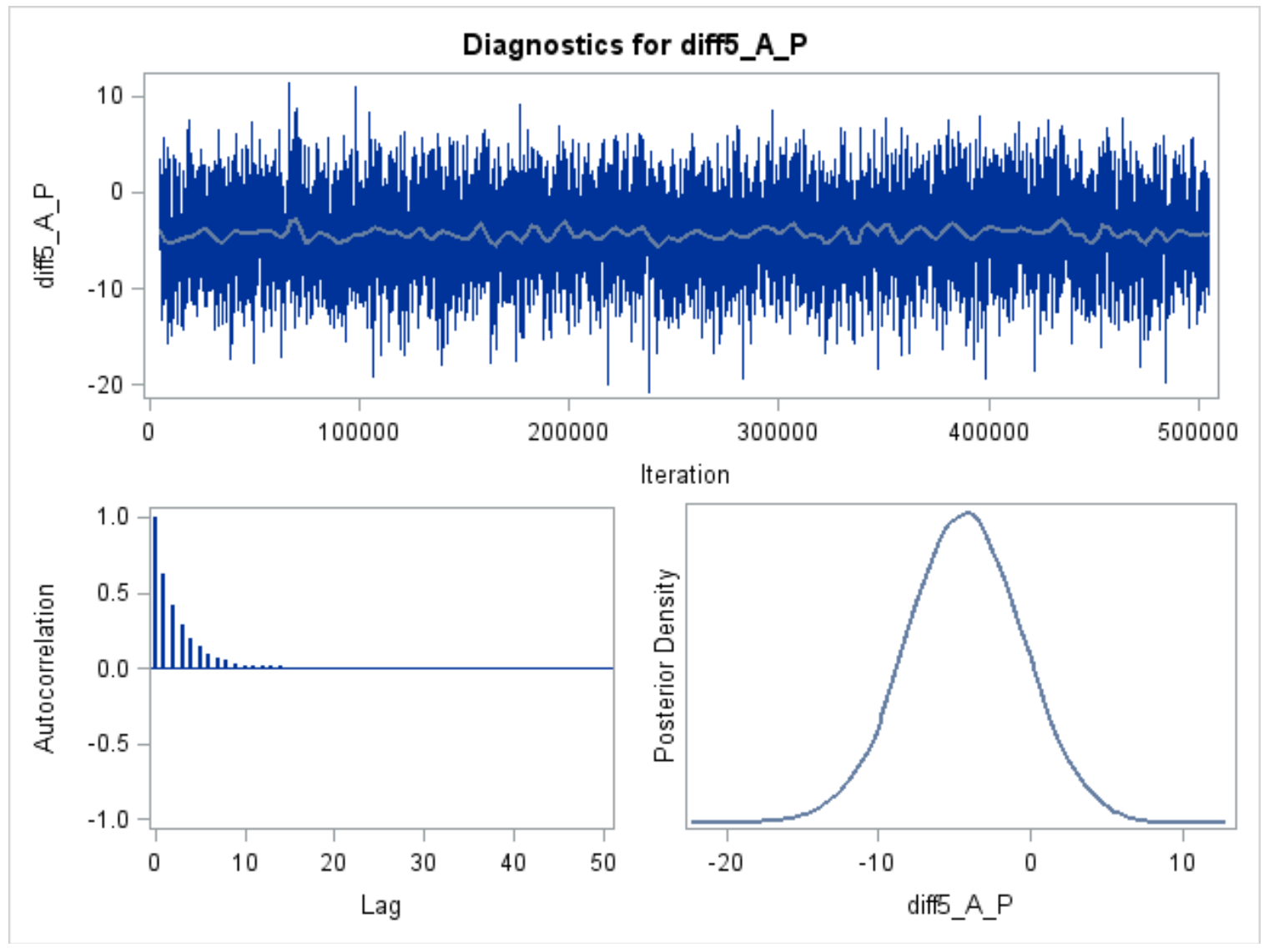




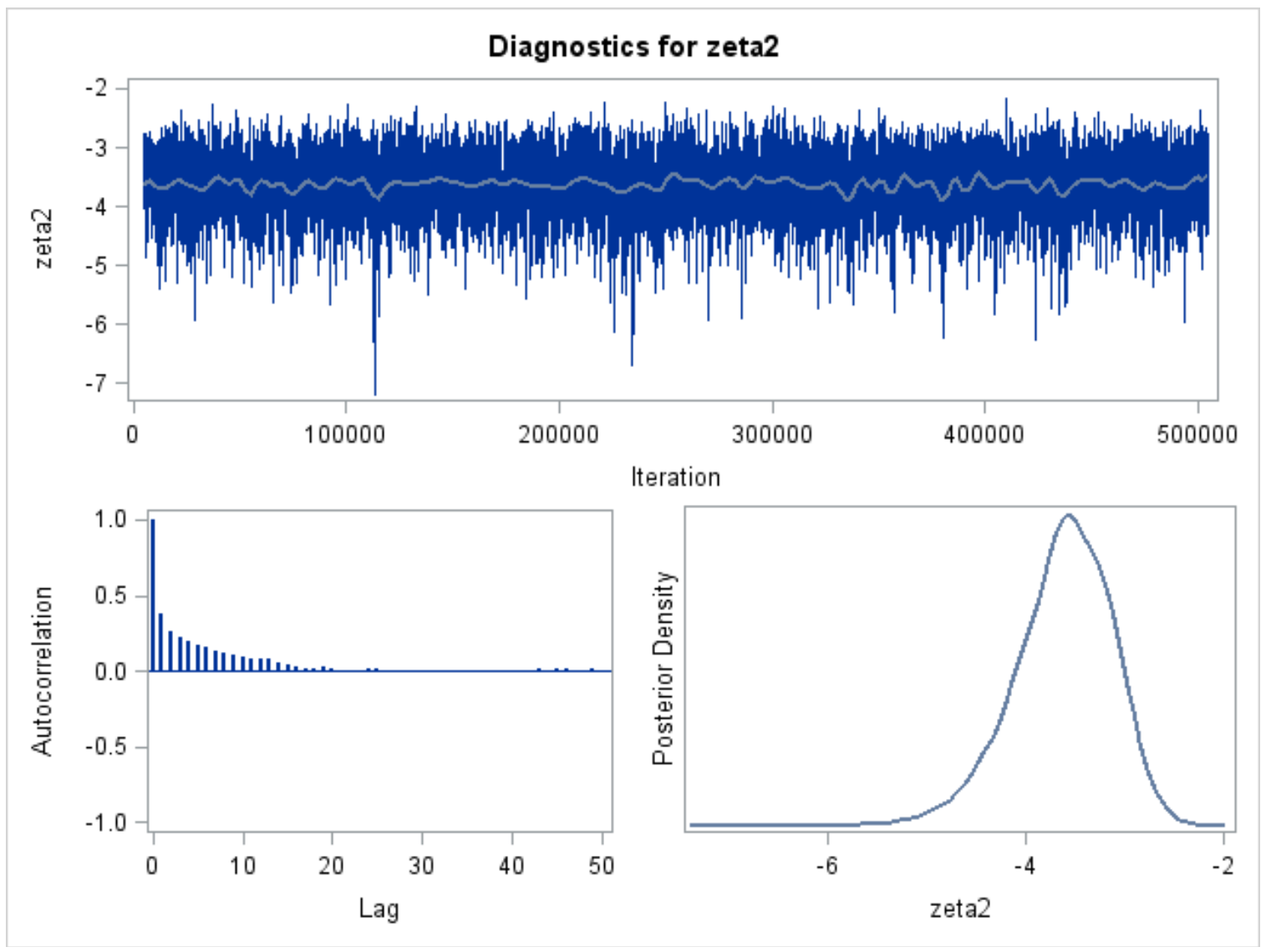




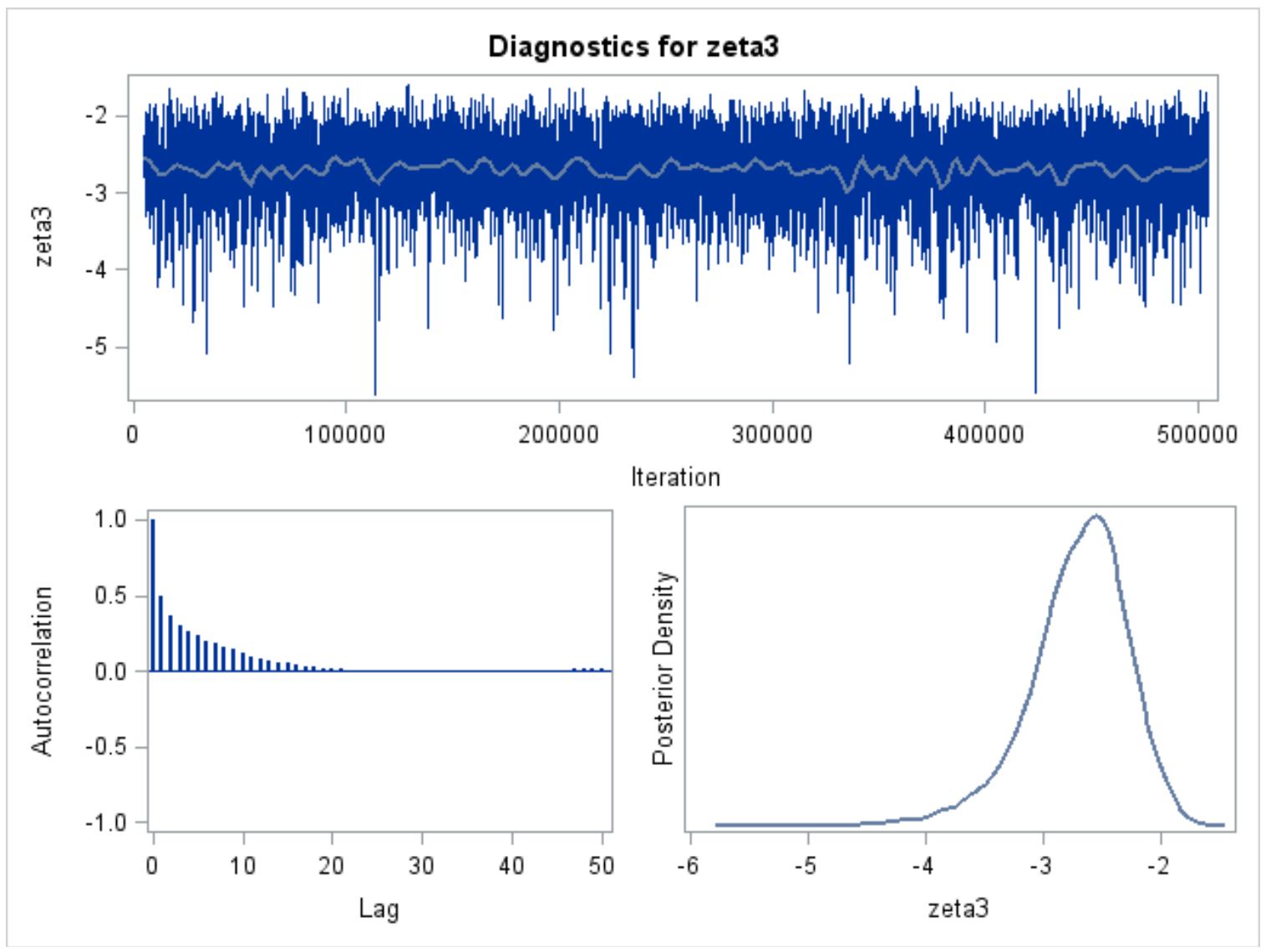




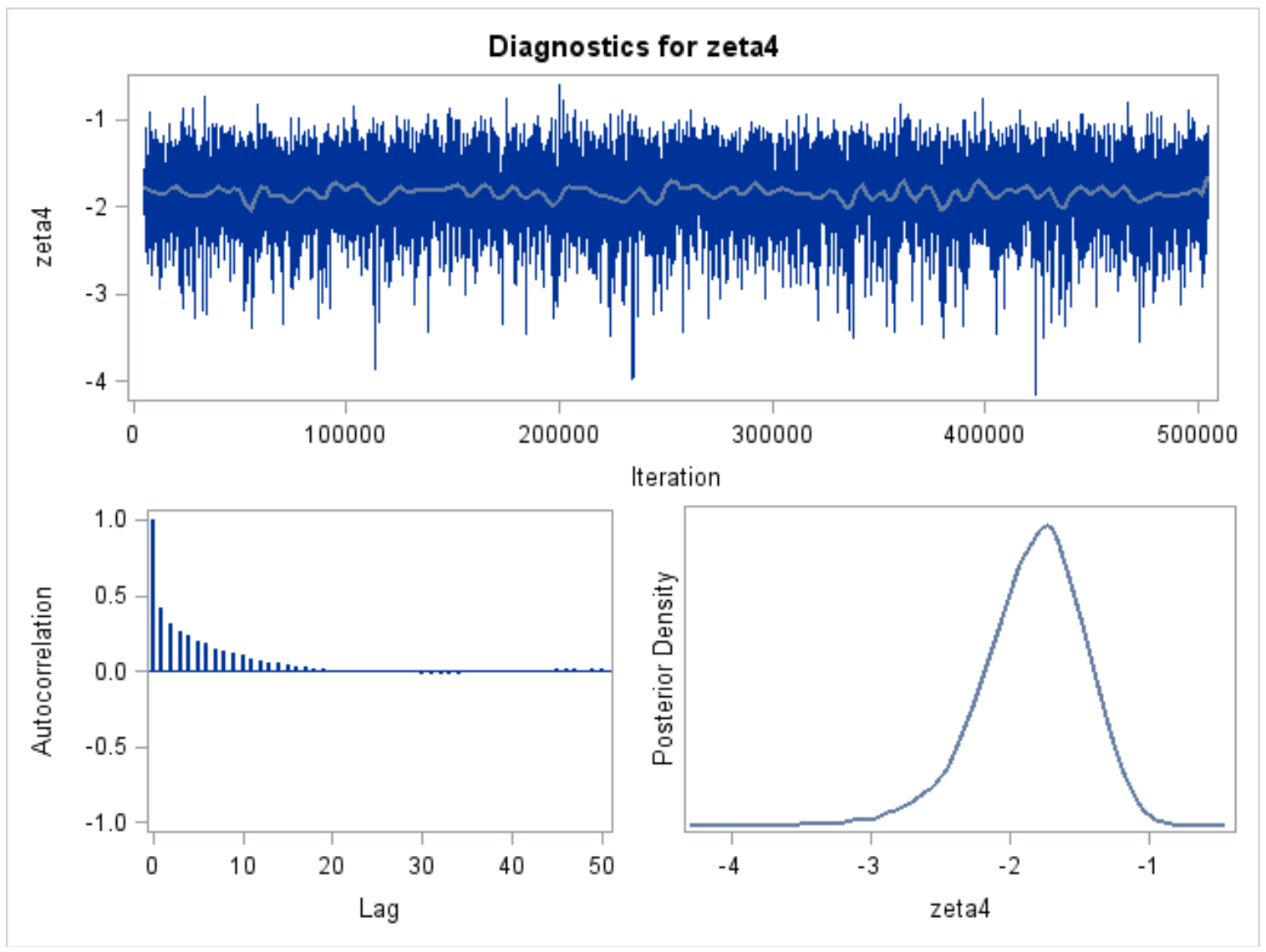




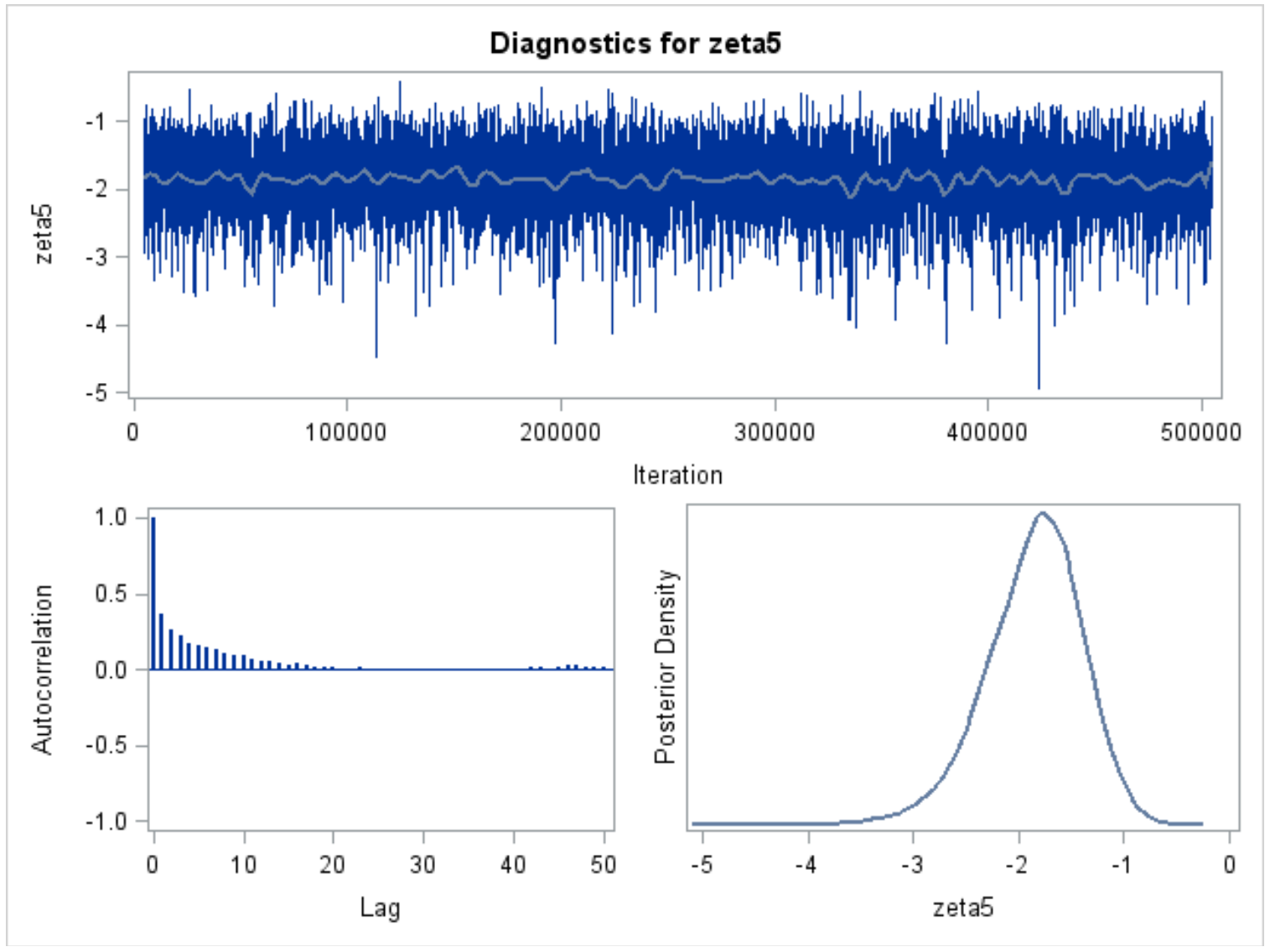




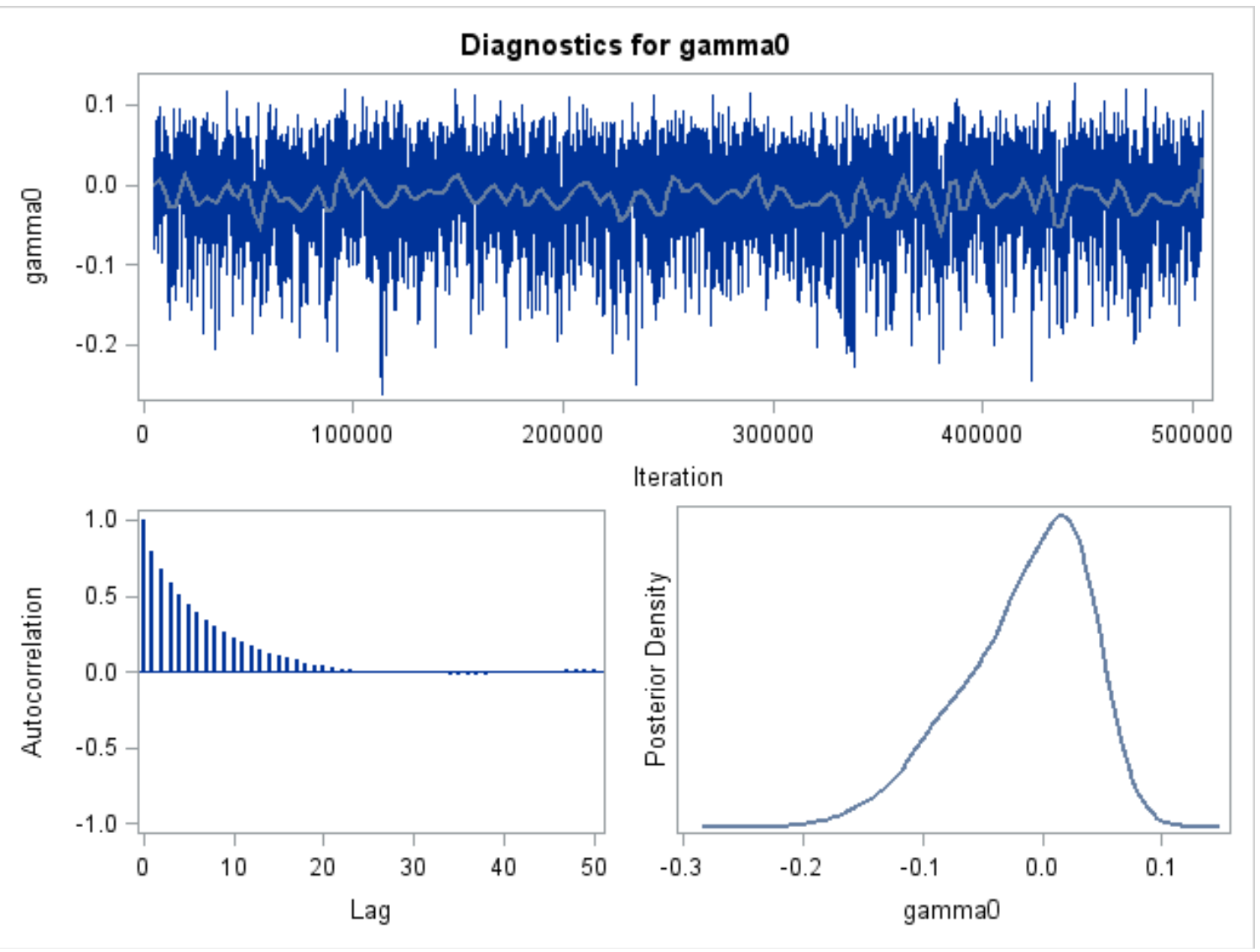




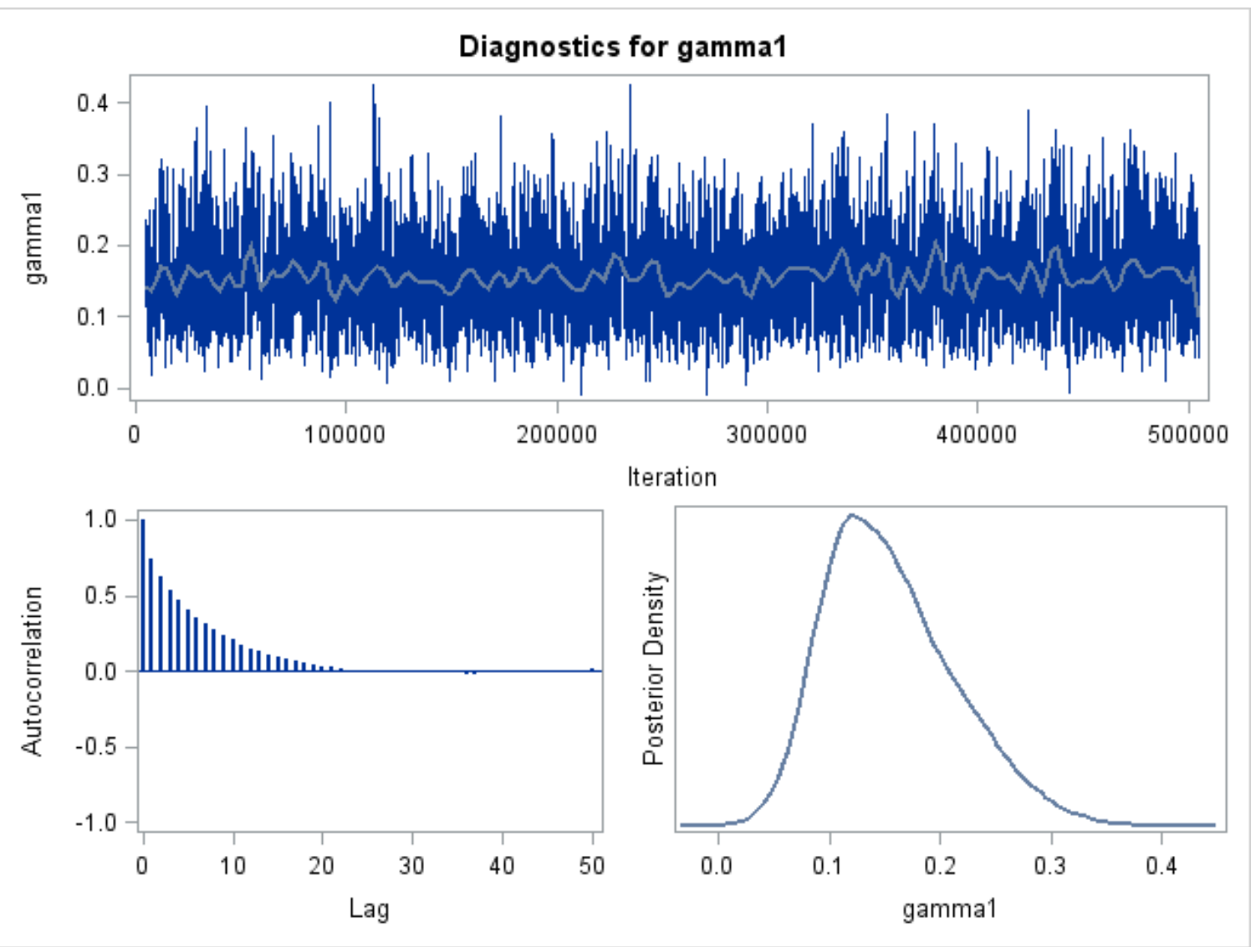


b). Trace and density plots from $4 \mathrm{MCMC}$ chains on model parameters from the Bayesian selection models (based on $\mathrm{R}$ and Stan codes as described in (d) above). The plot was produced using R coda packages. 4 different MCMC chains are run with 1000 burn-in and 5000 posterior samples.

Trace of alpha[1]

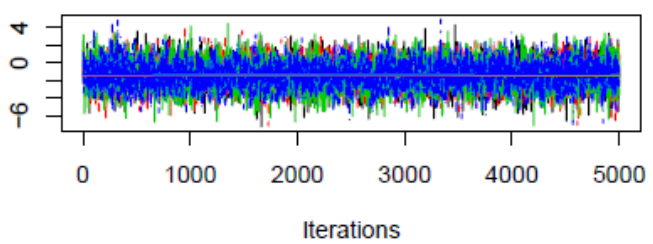

Trace of alpha[2]

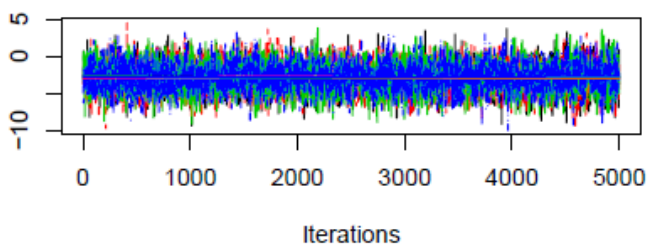

Trace of alpha[3]

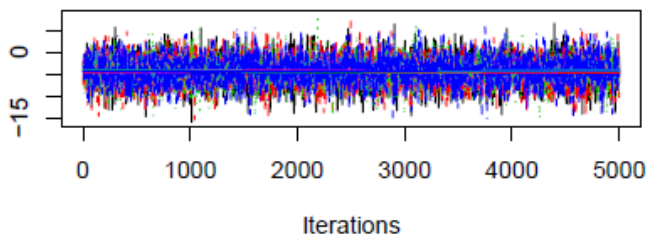

Trace of alpha[4]

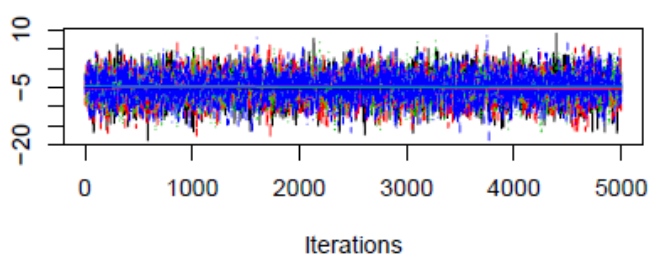

Density of alpha[1]

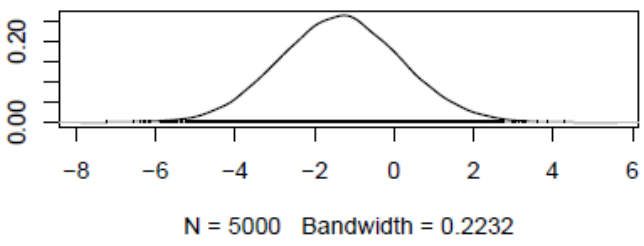

Density of alpha[2]

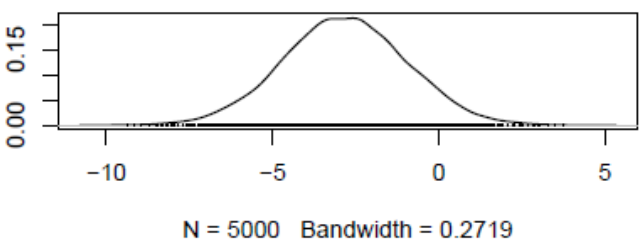

Density of alpha[3]

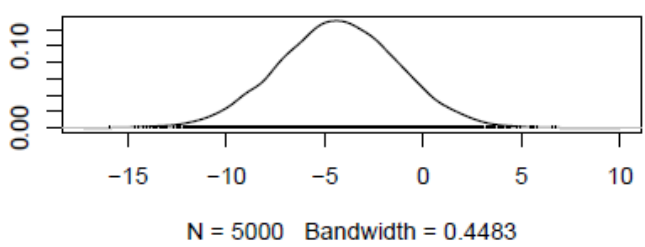

Density of alpha[4]

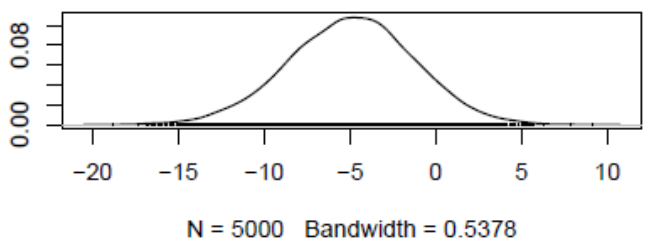


Trace of alpha[5]

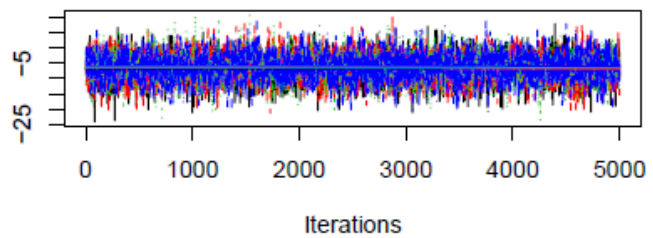

Trace of contrast 1

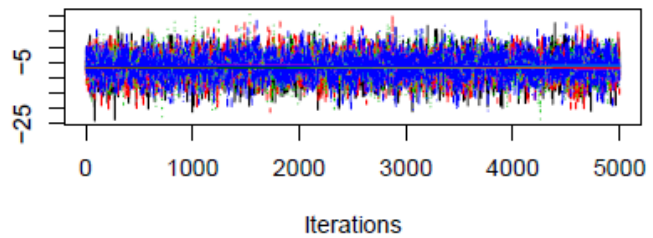

Trace of psi0[1]

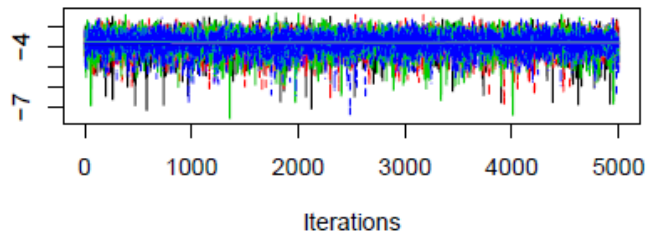

Trace of psi0[2]

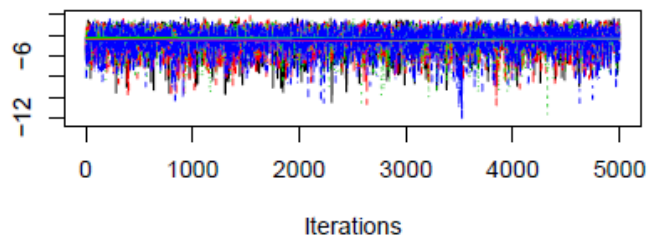

Density of alpha[5]

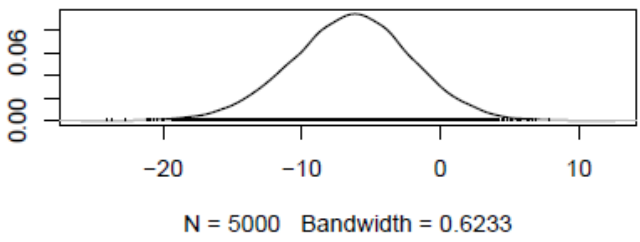

Density of contrast1

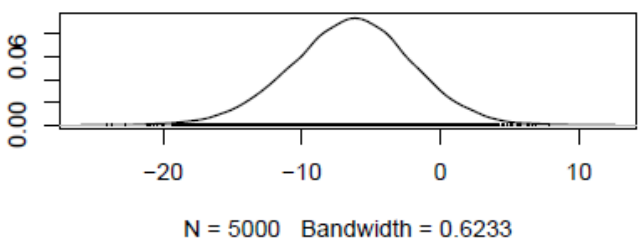

Density of psi0[1]

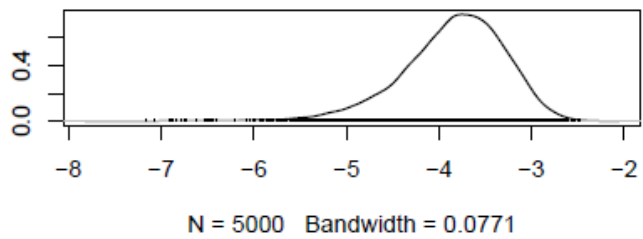

Density of psi0[2]

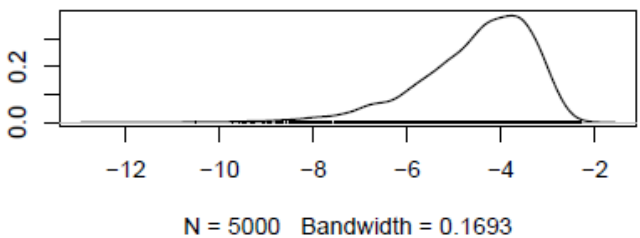


Trace of psi1[1]

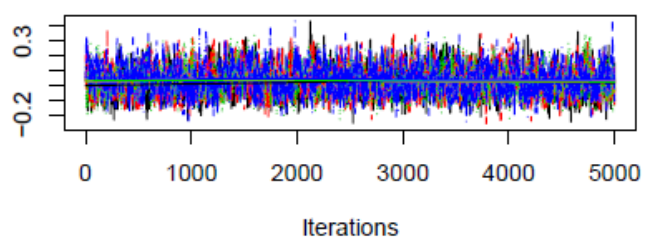

Trace of psi1[2]

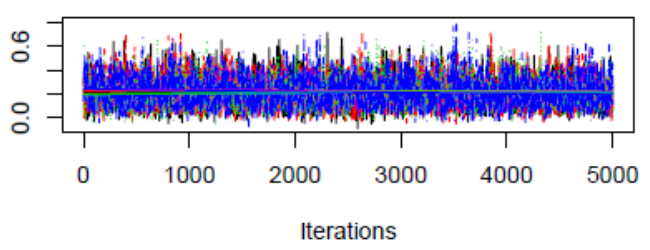

Trace of psi2[1]

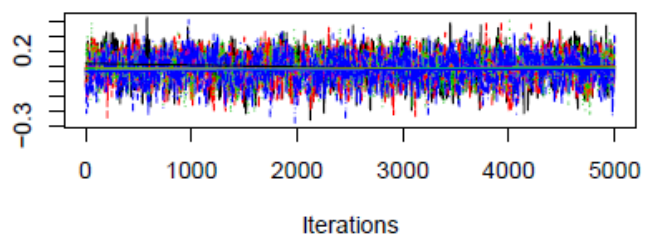

Trace of psi2[2]

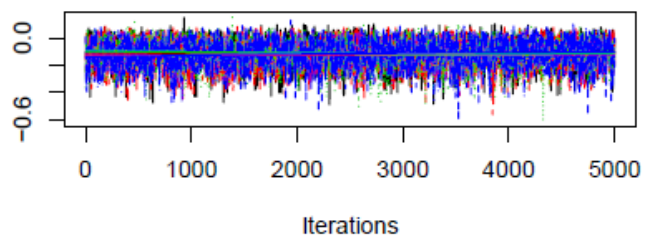

Density of psi1[1]

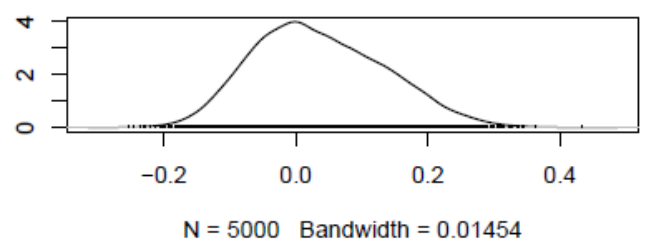

Density of psi1 [2]

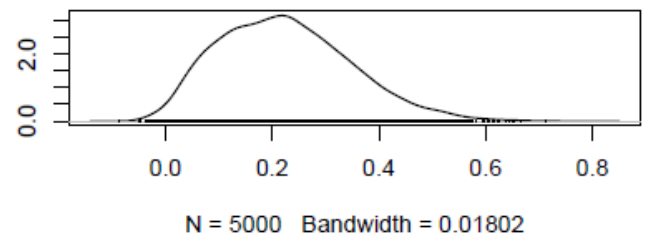

Density of psi2[1]

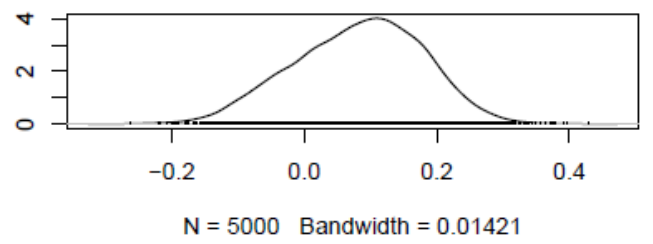

Density of psi2[2]

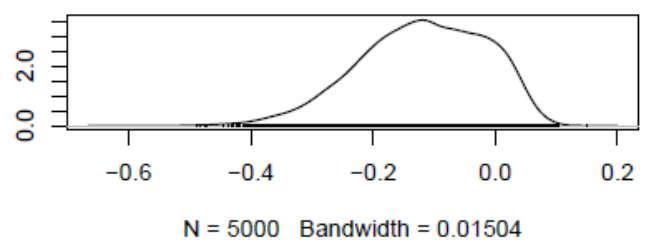


Trace of gam[1]

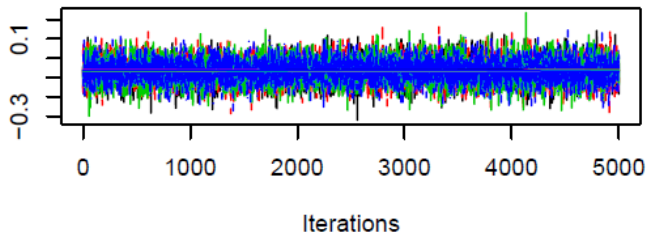

Trace of gam[2]

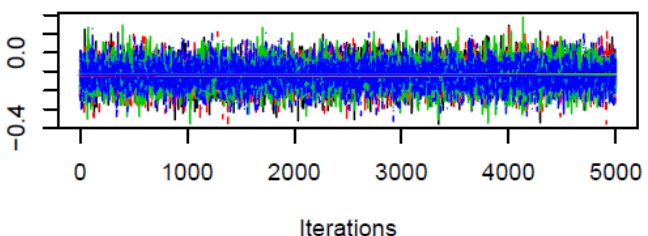

Trace of gam[3]

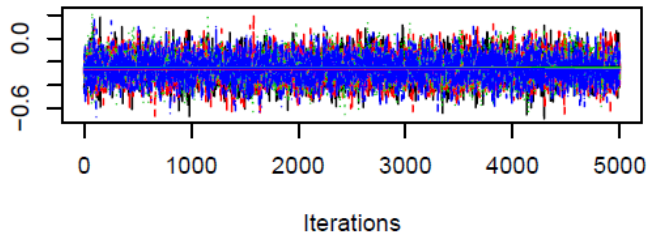

Trace of gam[4]

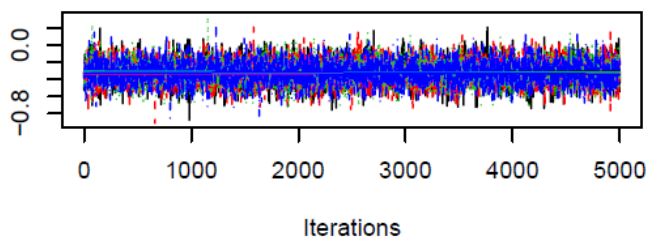

Density of gam[1]

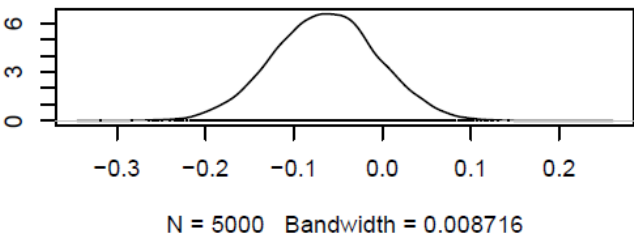

Density of gam[2]

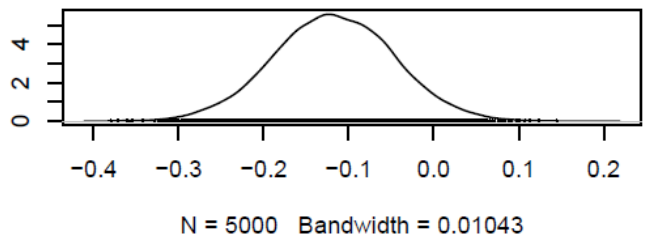

Density of gam[3]

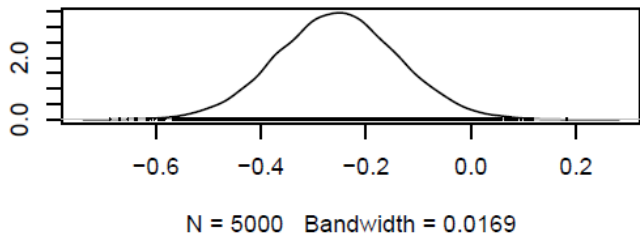

Density of gam[4]

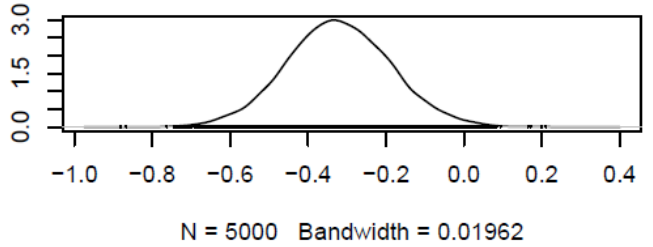


Trace of gam[5]

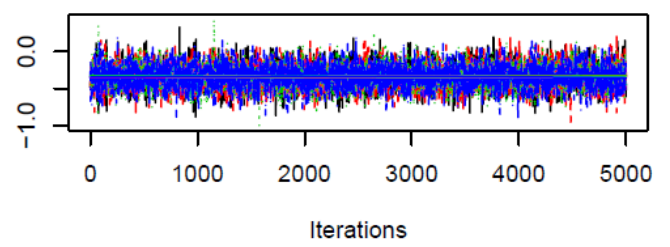

Trace of Sigma[1,1]

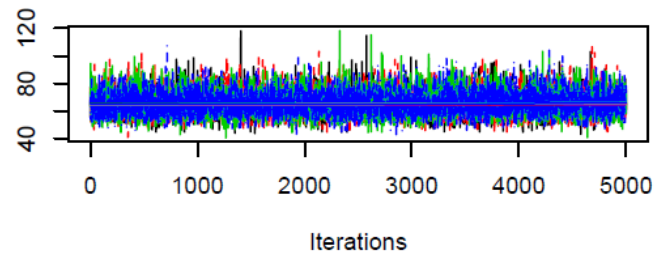

Trace of Sigma[1,2]

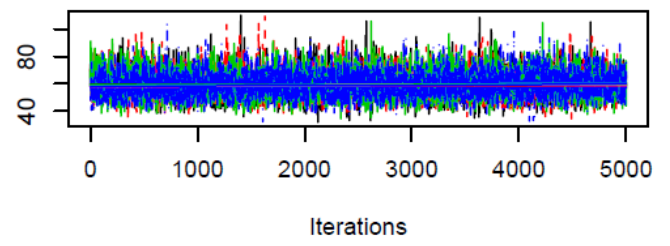

Trace of Sigma[1,3]

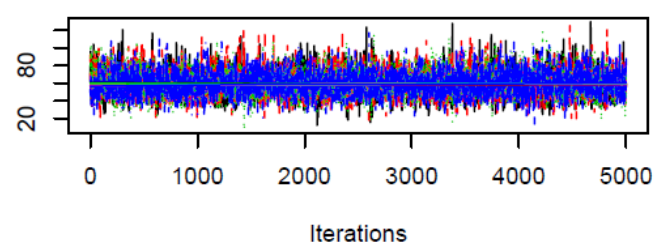

Density of gam[5]

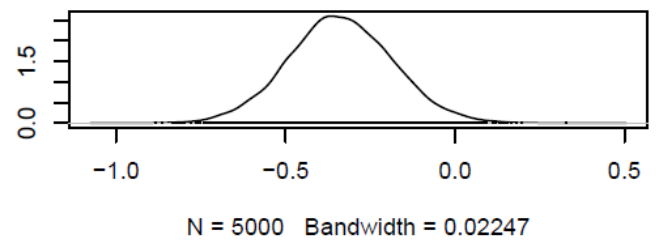

Density of Sigma[1,1]

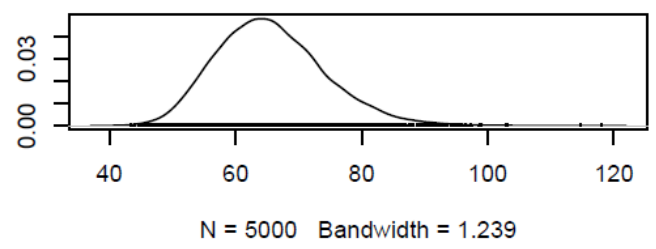

Density of Sigma[1,2]

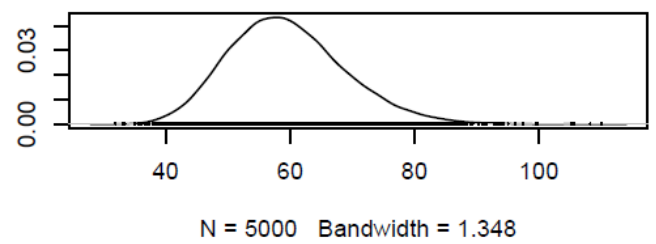

Density of Sigma[1,3]

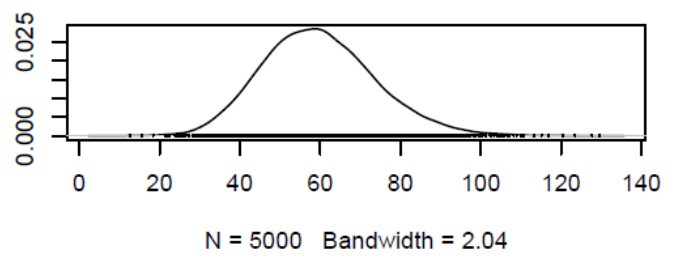


Trace of Sigma[1,4]

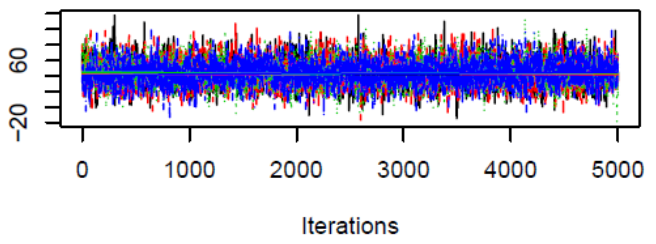

Trace of Sigma[1,5]

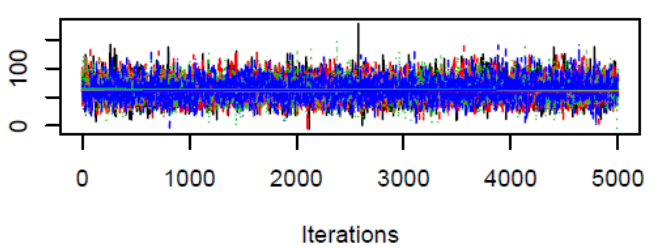

Trace of Sigma[2,1]

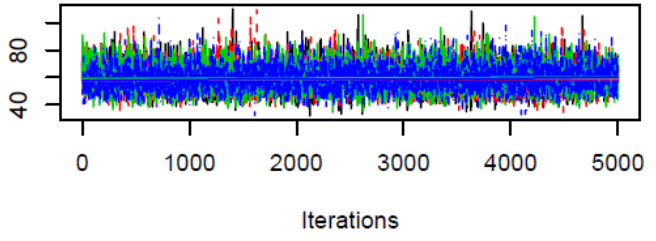

Trace of Sigma[2,2]

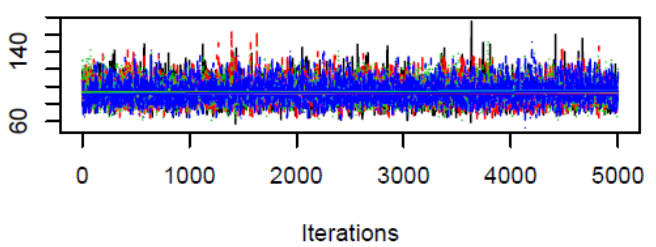

Density of Sigma[1,4]

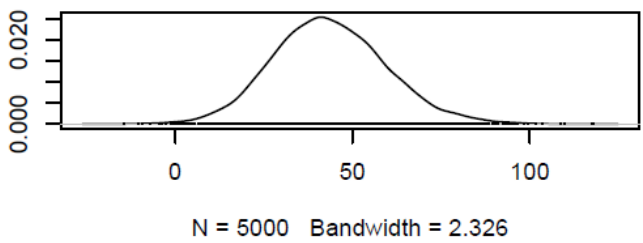

Density of Sigma[1,5]

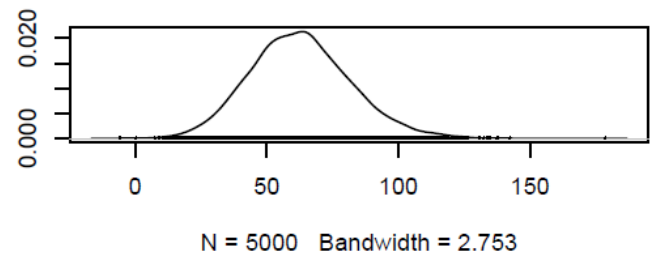

Density of Sigma[2,1]

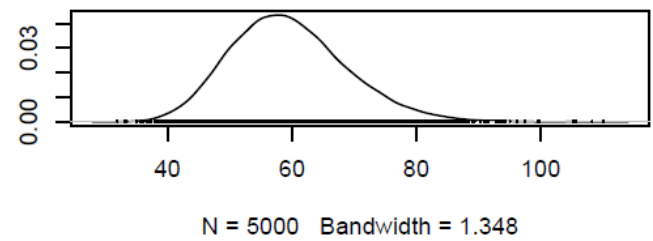

Density of Sigma[2,2]

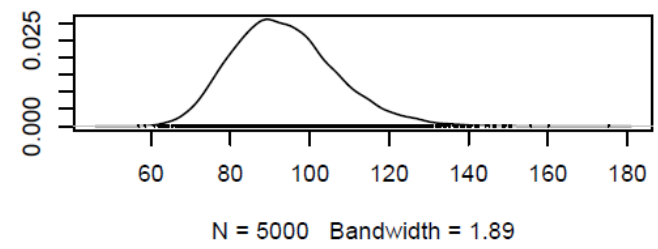

